Source: Gholamali, H., Shafiekhani, A., Darabi, E., \& Elahi, S. M. (2018). Ag/Au Alloy LSPR Engineering by Codeposition of RF-Sputtering and RF-PECVD. Applied Organometallic Chemistry, 32(5). https://doi.org/10.1002/aoc.4316

\title{
Ag/Au Alloy LSPR Engineering by Co-deposition of RF- Sputtering and RF-PECVD
}

\author{
Hediyeh Gholamali ${ }^{1}$, Azizollah Shafiekhani ${ }^{2,3}$,Elham Darabi ${ }^{1}$, Seyed Mohammad Elahi $^{1}$
}

${ }^{1}$ Department of Physics, Science and Research Branch, Islamic Azad University, Tehran, Iran.

${ }^{2}$ Department of Physics, Faculty of Physics and Chemistry, Alzahra University, Vanak, Tehran, Iran.

${ }^{3}$ School of Physics, Institute for Research in Fundamental Sciences, P.O. Box 19395-5531, Tehran, Iran.

\begin{abstract}
Silver-Gold alloy/diamond like carbon (Ag-Au/DLC) nanocomposite films were prepared by co-deposition of RF-sputtering and RF-PECVD on glass substrates by using acetylene gas and silver-gold target. The deposition process was carried out at room temperature in one minute with the variable parameters of initial pressures and $\mathrm{RF}$ powers. X-ray diffraction analysis demonstrated the formation of $\mathrm{Ag} / \mathrm{Au}$ alloy nanoparticles with a face-centered cubic (FCC) structure. Localized surface plasmon and optical properties of $\mathrm{Ag}$-Au alloy nanoparticles were studied by UV-visible spectrophotometry which showed that increasing RF power and initial pressure cause a redshift in all samples. Moreover, the effect of RF power and initial pressure on the size and shape of nanoparticles were studied by 2D Atomic force microscopy images. Energy dispersive X-ray spectroscopy revealed the formation of $\mathrm{Ag}$ $\mathrm{Au} / \mathrm{DLC}$ nanoparticles and the percentages of $\mathrm{C}, \mathrm{Ag}, \mathrm{Au}$ and $\mathrm{O}$ in all samples. The applied method for $\mathrm{Ag} / \mathrm{Au}$ alloy preparation is the one step and low-cost method which makes the samples ready for sensing application.
\end{abstract}

Keywords: AFM, Ag-Au alloy/DLC nanoparticles, LSPR, Optical properties, RF-sputtering and RF-PECVD. 